\title{
ON $k$-FREE INTEGERS WITH SMALL PRIME FACTORS
}

\section{G. HAZLEWOOD}

ABSTRACT. The object of this note is to give a nontrivial lower estimate for the function $\psi_{k}(x, y ; h)$, defined to be the number of $k$-free integers $m$ such that $1 \leq m<x,(m, h)=1$, and $m$ has no prime factor greater than or equal to $y$.

Let $k \geq 2$ be a fixed natural number and let $\mu_{k}(n)$ denote the multiplicative function given for powers of an arbitrary prime $p$ by

$$
\mu_{k}\left(p^{a}\right)=\left\{\begin{aligned}
1, & a=0 \\
-1, & a=k \\
0, & \text { otherwise. }
\end{aligned}\right.
$$

Let $h$ be a natural number, $x>0, y \geq 2$ be real numbers and let $f_{k}(n)=$ $\Sigma_{d \mid n} \mu_{k}(d)$. Let $p(n)$ denote the largest prime factor of $n$ with $p(1)=1$. Then the sum

$$
\psi_{k}(x, y ; h)=\sum_{n<x ; p(n)<y ;(n, h)=1} f_{k}(n)
$$

denotes the number of $k$-free (having no $k$ th power divisors) natural numbers less than $x$, relatively prime to $h$, and free of prime factors greater than or equal to $y$.

Now we let $t$ be a real number such that $x=y^{t}$ and $t=\log x / \log y$. The purpose of this note is to present a lower bound for $\psi_{k}\left(y^{t}, y ; h\right)$, using only elementary methods, which has very few restrictions on $y, t$, and $h$. that

Theorem. Let $t \geq 3$. Given $t$, there exists a real number $y_{1}=y_{1}(t)$ such

$$
\psi_{k}\left(y^{t}, y ; h\right) \geq 2 e^{-10} \prod_{p \mid h}\left(\frac{1-p^{-1}}{1-p^{-k}}\right) \frac{y^{t}}{\zeta(k)} \exp \{-t(\log t+\log \log t+\eta(t))\}
$$

for each $y \geq y_{1}$ and $h \leq(\log y)^{C}$ for some absolute constant $C$, where

Received by the editors May 13, 1974 and, in revised form, July 15, 1974. AMS (MOS) subject classifications (1970). Primary 10-02, 10H15, $10 \mathrm{H} 20$.

Key words and phrases. Numbers with small prime factors, $k$-free integers, Buchstab identity. 


$$
\eta(t)=\frac{1}{\log t}\left(\log \log t+1-\frac{\log \log t}{\log t}+\frac{4}{\log t}+\frac{4 \log t}{t}\right)
$$

and $\zeta(k)$ is Riemann's zeta function.

For comparison, we note that as a consequence of the remark on p. 199 and Theorem 3.2.4 of Levin and Fainleib [5]

$$
\lim _{y \rightarrow \infty} \frac{\psi_{k}\left(y^{t}, y ; h\right)}{\prod_{p \mid h}\left(\frac{1-p^{-1}}{1-p^{-k}}\right) \frac{y^{t}}{\zeta(k)} Z(t)}=1
$$

for $t \leq(\log y)^{3 / 5-\delta}, \delta>0, h \leq(\log y)^{C}, C$ an absolute constant, where $Z(t)$ satisfies de Bruijn's differential-difference equation $t Z^{\prime}(t)=-Z(t-1)$ with initial condition $Z(t)=1$ for $0 \leq t \leq 1$. In particular,

$$
Z(t)=\exp \{-t(\log t+\log \log t+o(1))\}
$$

as $t$ approaches $\infty$.

We can also state a comparable upper bound with the restricted range, $e<t<y / e \log y, h \leq y$, using the proof of Theorem 3 of [4] with $g(n)=f_{k}(n) / n$ :

$$
\begin{aligned}
& \psi_{k}\left(y^{t}, y ; h\right) \\
& \quad \leq \prod_{p \mid b}\left(\frac{1-p^{-1}}{1-p^{-k}}\right) \prod_{p<y}\left(1-p^{-k}\right) y^{t} \exp \{-t \log t-t \log \log t+\eta(t, y)\}
\end{aligned}
$$

where

$$
\eta(t, y)=t\left\{1-\frac{\log \log t}{\log t}+O\left(\frac{1}{\log t}\right)\right\}+O(\log \log y)+O\left(\frac{(t \log t)^{2}}{y \log y}\right) .
$$

Throughout the discussion, the constants implied by the use of the $O$-notation are absolute.

In order to prove the Theorem, we use essentially the method of Halberstam [1] and [3] together with an estimate for $Q_{k}(x ; h)$, the number of $k$-free natural numbers less than $x$ that are relatively prime to $h$, and a generalization of the Buchstab identity.

We note that

$$
Q_{k}(x ; h)=\frac{x}{\zeta(k)} \prod_{p \mid h}\left(\frac{1-p^{-1}}{1-p^{-k}}\right)+O\left(x^{1 / k} \frac{\phi(h)}{h} \quad 2^{\nu(h)}\right)+O\left(4^{\nu(h)}\right)
$$

where $\phi(h)$ is Euler's totient function and $\nu(h)$ denotes the number of distinct prime factors of $h$, by the following argument: 
Using the first line of the proof of Theorem 3.1 in Harris and Subbarao [2]

$$
Q_{k}(x, h)=\sum_{d<x ;(d, h)=1} \mu_{k}(d) \sum_{n<x / d ;(n, h)=1} 1
$$

so that

$$
Q_{k}(x ; h)=\sum_{d<x ;(d, h)=1} \mu_{k}(d)\left\{\frac{\phi(h)}{h} \frac{x}{d}+O\left(2^{\nu(h)}\right)\right\}
$$

The leading term of (8) follows from the Harris-Subbarao argument. The error term

$$
\begin{aligned}
& =O\left(2^{\nu(h)} \sum_{d<x ;(d, h)=1} \mu_{k}^{2}(d)\right)=O\left(2^{\nu(h)} \sum_{d<x^{1 / k} ;(d, h)=1} 1\right) \\
& =O\left(2^{\nu(h)}\left\{\frac{\phi(h)}{h} x^{1 / k}+O\left(2^{\nu(h)}\right)\right\}\right)=O\left(2^{\nu(h)} \frac{\phi(h)}{h} x^{1 / k}\right)+O\left(4^{\nu(h)}\right) .
\end{aligned}
$$

We alsu remark that if $0<x \leq y$, then

$$
\psi_{k}(x, y ; h)=Q_{k}(x ; h) .
$$

As for the generalization of the Buchstab identity, we let $A$ denote a nonempty finite set of natural numbers and define

$$
G(A, y, g)=\sum_{a \in A ; p(a)<y} g(a), \quad g \text { multiplicative }
$$

then

(11) $G(A, u, g)-G(A, v, g)=\left\{\begin{array}{l}\Sigma_{v \leq p<u} \Sigma_{m=1}^{\infty} g\left(p^{m}\right) G\left(A_{p}, p, g\right), \quad 2 \leq v \leq u, \\ 0, \quad \text { otherwise, }\end{array}\right.$ where

$$
A_{p^{m}}=\left\{\frac{a}{p^{m}} \mid a \in A, a \equiv 0\left(\bmod p^{m}\right), a \equiv 0\left(\bmod p^{m+1}\right)\right\} .
$$

In view of [1] and [3], we will omit the proof of (11).

Now we state the first of 2 lemmas needed to prove the Theorem.

Lemma 1. If $t$ is a real number such that $1<t \leq 2$ and $h$ is a natural number, then

$$
\psi_{k}\left(y^{t}, y ; h\right) \geq \prod_{p \mid h}\left(\frac{1-p^{-1}}{1-p^{-k}}\right) \frac{y^{t}}{\zeta(k)}\left\{1-\log t+o\left(\frac{4^{\nu(b)} \log \log 3 h}{\log y}\right)\right\} .
$$

Proof. We take $A=\left\{n \mid 1 \leq n<y^{t},(n, h)=1\right\}, u=y^{t}, v=y$, and $g(n)=$ $f_{k}(n)$ in (11) so that by (8) and (9), we have

$$
\begin{aligned}
\psi_{k}\left(y^{t}, y ; h\right)= & \prod_{p \mid h}\left(\frac{1-p^{-1}}{1-p^{-k}}\right) \frac{y^{t}}{\zeta(k)}+O\left(y^{t / k} \frac{\phi(h)}{h} 2^{\nu(h)}\right) \\
& +O\left(4^{\nu(h)}\right)-\sum_{y \leq p<y^{t} ; p \nmid b} Q_{k}\left(\frac{y^{t}}{p} ; h\right),
\end{aligned}
$$


since $y \leq p<y^{t}$ and $1<t \leq 2$ imply $y^{t} / p \leq p$.

Hence

$\psi_{k}\left(y^{t}, y ; h\right)=\prod_{p \mid h}\left(\frac{1-p^{-1}}{1-p^{-k}}\right) \frac{y^{t}}{\zeta(k)}\left\{1-\sum_{y \leq p<y^{t} ; p \nmid h} \frac{1}{p}\right\}+O\left(y^{t / k} \frac{\phi(h)}{h} 2^{\nu(h)}\right)$

$$
\begin{aligned}
& +O\left(4^{\nu(h)}\right)+O\left(y^{t / k} \frac{\phi(h)}{h} 2^{\nu(h)} \sum_{y \leq p<y^{t} ; p \nmid h} p^{-1 / k}\right)+O\left(4^{\nu(h)} \pi\left(y^{t}\right)\right) \\
& =\prod_{p \mid h}\left(\frac{1-p^{-1}}{1-p^{-k}}\right) \frac{y^{t}}{\zeta(k)}\left\{1-\sum_{y \leq p<y^{t} ; p \nmid h^{p}} \frac{1}{p}\right\}+O\left(4^{\nu(h)} \frac{y^{t}}{\log y}\right) .
\end{aligned}
$$

Now, as in [3], we observe that

$$
\sum_{p<x ; p \nmid h} \frac{1}{p}=\log \log x+\left(B-\sum_{p \mid h} \frac{1}{p}\right)+O\left(\frac{1}{\log x}+\frac{\nu(h)}{x}\right) \text {. }
$$

Thus with $1<t \leq 2$

$$
\begin{aligned}
\psi_{k}\left(y^{t}, y ; h\right) & =\prod_{p \mid h}\left(\frac{1-p^{-1}}{1-p^{-k}}\right) \frac{y^{t}}{\zeta(k)}\left\{1-\log t+O\left(\frac{1}{\log y}+\frac{\nu(h)}{y}+\frac{4^{\nu(h)} \log \log 3 b}{\log y}\right)\right\} \\
& =\prod_{p \mid h}\left(\frac{1-p^{-1}}{1-p^{-k}}\right) \frac{y^{t}}{\zeta(k)}\left\{1-\log t+O\left(\frac{4^{\nu(h)} \log \log 3 h}{\log y}\right)\right\},
\end{aligned}
$$

to complete the proof of the lemma.

Due to the factor $4^{\nu(h)}$ in the error term, we are forced to restrict $h$ by the condition

$$
h \leq(\log y)^{C}
$$

for an absolute constant $C$.

Now we define

$$
\Lambda_{k}(x, y ; h)=\left(\prod_{p \mid h}\left(\frac{1-p^{-1}}{1-p^{-k}}\right) \frac{y^{t}}{\zeta(k)}\right)^{-1} \psi_{k}(x, y ; h)
$$

and state Lemma 2, whose proof is almost identical to that of Lemma 3 of [3] and so will be omitted.

Lemma 2. Let $\delta$ be a number satisfying

$$
0<\delta<1 / 3 \text {. }
$$

For each natural number $n$, there exists a number $y_{1}=y_{1}(n, \delta)$ such that if $1 / 3<t \leq n(1-\delta)$, then

$$
\Lambda_{k}\left(y^{t}, y ; h\right) \geq(2 / n !) \delta^{n-1}
$$

for $y \geq y_{1}$ and $h \leq(\log y)^{C}$ where $C$ is an absolute constant.

The proof of the Theorem follows from Lemma 2 by a suitable choice of 
$n$ and $\delta$ in terms of $t$, namely $n=[(t+1) /(1-\delta)]$ and $\delta=1 /(2+\log (t+1))$ (see Halberstam [1]).

\section{REFERENCES}

1. H. Halberstam, On integers all of whose prime factors are small, Proc. London Math. Soc. (3) 21 (1970), 102-107. MR $42 \# 4509$.

2. V. C. Harris and M. V. Subbarao, An arithmetic sum with an application to quasi-k-free integers, J. Austral. Math. Soc. 15 (1973), 272-278. MR 48 \#8363.

3. D. G. Hazlewood, On integers all of whose prime factors are small, Bull. London Math. Soc. 5 (1973), 159-163.

4. - Sums over positive integers with few prime factors, J. Number Theory 7 (1975), 189-207.

5. B. V. Levin and A. S. Fainleib, Application of certain integral equations to questions of the theory of numbers, Uspehi Mat. Nauk 22 (1967), no. 3 (135), 119-197 = Russian Math. Surveys 22 (1967), no. 3, 119-204. MR 37 \#174.

DEPARTMENT OF MATHEMATICS, SOUTHWEST TEXAS STATE UNIVERSITY, SAN MARCOS, TEXAS 78666 